'Departamento de Ciencias Neurológicas Oriente, Facultad de Medicina, Universidad de Chile. Santiago, Chile.

${ }^{2}$ Servicio de Neurología, Hospital San Camilo. San Felipe, Chile. ${ }^{3}$ Servicio de Neurorradiología, Instituto de Neurocirugía Dr. Asenjo. Santiago, Chile. ${ }^{4}$ Servicio de Neurología, Hospital del Salvador. Santiago, Chile.

Trabajo no recibió financiamiento. Los autores declaran no tener conflictos de interés.

Recibido el 17 de enero de 2019, aceptado el 23 de mayo de 2019 .

Correspondencia a: Dr. Gabriel Cea jcea@med.uchile.cl

\section{Xantomatosis cerebrotendinosa sin xantomas tendíneos. Caso clínico}

\author{
JUAN P. CONTRERAS ${ }^{1}$, GONZALO GUAJARDO², ARTURO MARTÍNEZ ${ }^{2}$, \\ INGEBORG LÓPEZ ${ }^{3}$, GABRIEL CEA ${ }^{1,4}$
}

\section{Cerebrotendinous xanthomatosis. Report of one case}

Cerebrotendinous xanthomatosis (CTX) is an uncommon autosomal recessive disease caused by deficiency of 27-sterol-hydroxylase that results in an accumulation of cholestanol in the central nervous system, eyes, tendons, and blood vessels. We report a 22-year-old woman with a history of cataract surgery at the age of 14, cholecystectomy due to cholelithiasis at the age of 17 and chronic diarrhea, who presented with a six months period of gait instability and frequent falls. Physical examination revealed a bilateral pyramidal and cerebellar syndrome, with no visible tendon xanthomas. Cerebral magnetic resonance imaging showed an increase of the signal intensity on the T2-weighted images in periventricular cerebral white matter, dentate nuclei and spinal cord. With a high suspicion of CXT, a genetic study was conducted identifying a pathogenic variant in the CYP27A1 gene. There is considerable variation in clinical characteristics and age of onset of this disease, including absence of tendon xanthomas, delaying the diagnosis. Early recognition and chronic chenodeoxycholic acid therapy can improve outcome and quality of life.

(Rev Med Chile 2019; 147: 658-662)

Key words: Chenodeoxycholic Acid; Cholestanol; Xanthomatosis; Xanthomatosis, Cerebrotendinous.

\section{L} a xantomatosis cerebrotendinosa (CXT) es una enfermedad autosómica recesiva causada por mutaciones en el gen CYP27A1, que codifica para la enzima esterol 27-hidroxilasa ${ }^{1}$. El defecto en esta enzima es responsable de una importante disminución del ácido quenodesoxicólico (CDCA) y aumento compensatorio de la actividad del colesterol 7- $\alpha$-hidroxilasa, lo que conduce a la acumulación de colestanol y otros precursores de ácidos biliares en plasma y tejidos ${ }^{2}$. La CXT puede comenzar durante la infancia o adultez, con progresión lenta y combinación de manifestaciones neurológicas y no neurológicas ${ }^{3}$. Se caracteriza por presentar diarrea y cataratas en la infancia, seguido de xantomas tendíneos y disfunción neurológica progresiva en la adultez (demencia, trastornos conductuales, signos piramidales, cerebelosos o ambos, distonía, parkinsonismo atípico, neuropatía periférica y convulsiones) ${ }^{4}$. Con menor frecuencia se observan alteraciones oftalmológicas del disco óptico y senescencia prematura de la retina ${ }^{5}$. Es importante destacar que los xantomas tendíneos rara vez se ven en pacientes menores de 20 años, por lo que el diagnóstico de CTX siempre debe ser considerado ante pacientes jóvenes con diarrea crónica y cataratas bilaterales ${ }^{6}$.

Presentamos el caso clínico de una paciente con xantomatosis cerebrotendinosa confirmada genéticamente sin xantomas tendíneos visibles, se discuten las dificultades que acompañan el diagnóstico y los beneficios del tratamiento precoz. 


\section{Caso clínico}

Paciente de sexo femenino, padres sanos no consanguíneos, hija mayor de tres hermanos de los cuales uno falleció a los dos días de vida por hernia diafragmática. Desarrollo psicomotor sin alteraciones. Antecedentes de cirugía de cataratas a los 15 años, colecistectomía por colelitiasis a los 17 años y diarrea crónica desde su infancia. Consultó a los 22 años por inestabilidad de la marcha y caídas frecuentes, dificultad para subir y bajar escaleras, desde al menos 6 meses. Al examen neurológico destacaba fuerza conservada en las cuatro extremidades, espasticidad, hiperreflexía incluido clonus, reflejo plantar extensor bilateral, marcha atáxica y dismetría. La sensibilidad estaba conservada en todas sus modalidades. Sin xantomas tendíneos visibles al examen físico.

El estudio por resonancia magnética (RM) de cerebro reveló lesiones hiperintensas en secuencias ponderadas en T2 (T2W) en ambos núcleos dentados y discreto incremento de señal de sustancia blanca periventricular posterior, sin restricción a la difusión y sin realce tras el uso de contraste (Figura 1). En la RM de médula se observaron extensas lesiones intramedulares desde C2 hasta D10, hiperintensas en secuencias ponderadas en T2, que comprometían predominantemente los cordones posteriores y laterales a ambos lados sin realce tras el uso de medio de contraste (Figura 2). Estudio de conducción nerviosa reveló una velocidad de conducción motora en límite normales y un estudio sensitivo normal en ambos nervios surales. La electromiografía no reveló signos de denervación activa ni crónica. En el resto de los exámenes destacaba lipoproteína de baja densidad (LDL) $61 \mathrm{mg} / \mathrm{dL}$, lipoproteína de alta densidad (HDL) $66 \mathrm{mg} / \mathrm{dL}$, triglicéridos $394 \mathrm{mg} / \mathrm{dL}$, acido láctico $31,1 \mathrm{mg} / \mathrm{dL}$, electrocardiograma (ECG) y ecocardiograma normales, y densitometría ósea que reveló osteopenia moderada en columna lumbar y huesos femorales. Además, se realizó evaluación neuropsicológica, donde se describió una eficiencia cognitiva global disminuida, con un coeficiente intelectual de 62 (WAIS IV), valores descriptivos cualitativos de discapacidad intelectual leve.

Ante la alta sospecha de CXT, se realizó la secuenciación del gen CYP27A1 mediante Next Generation Sequencing (NGS), en el Laboratorio

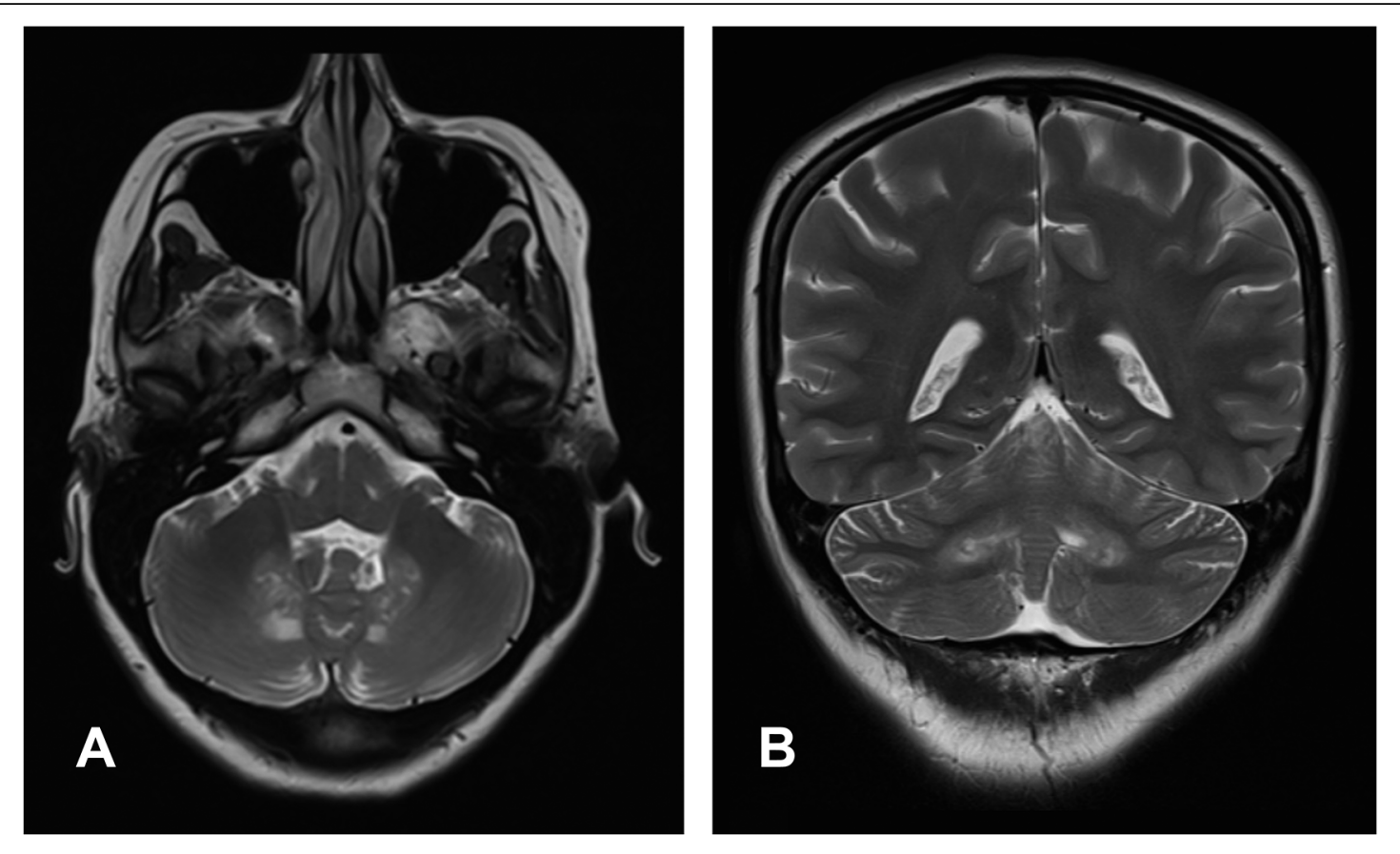

Figura 1. RM de encéfalo en $T 2 W$ axial (A) y coronal (B): Se observa incremento de señal en ambos núcleos dentados. 


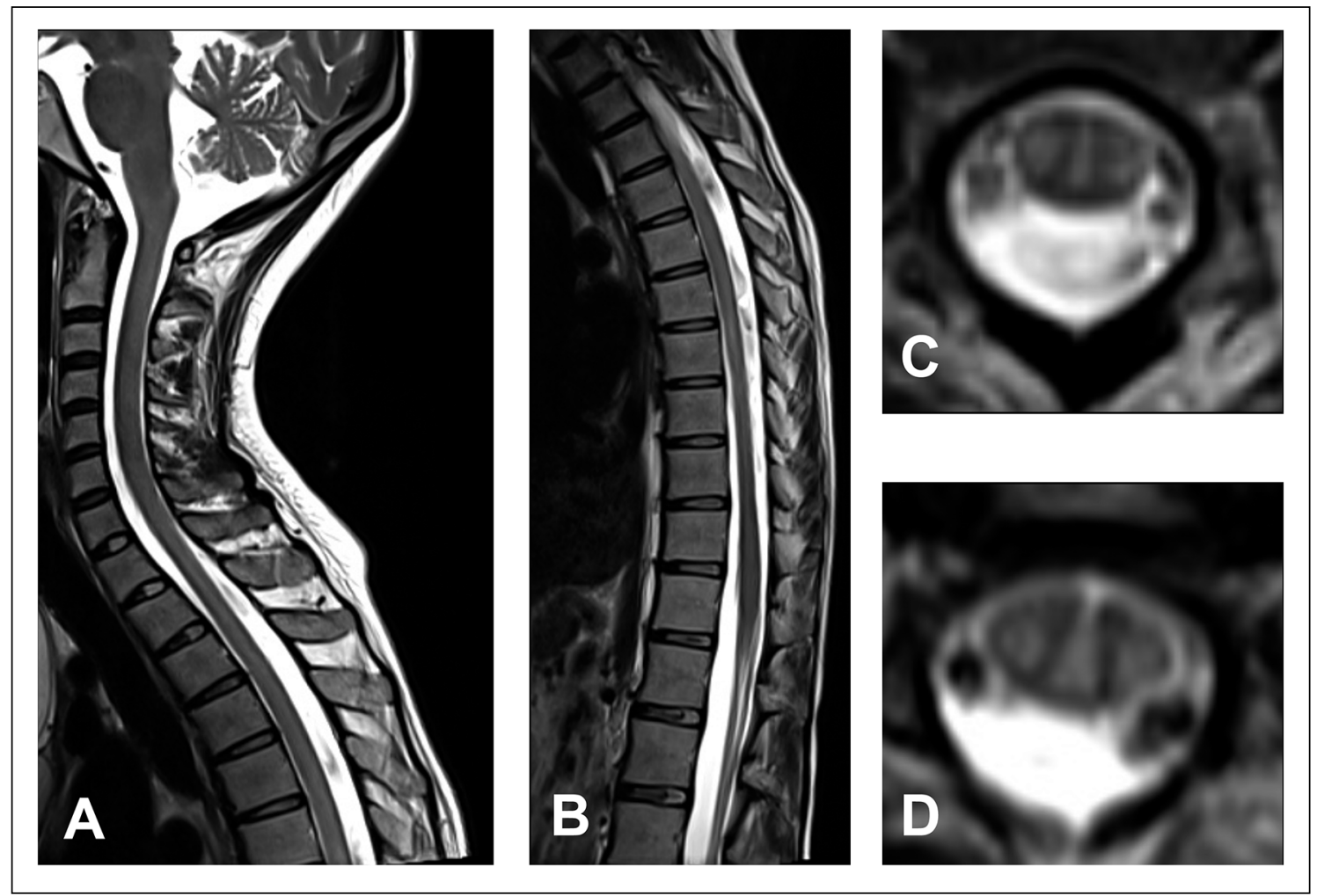

Figura 2. $R M$ de medula en $T 2 W$ sagital $(\mathbf{A}$ y $\mathbf{B})$ y axiales a nivel dorsal en $(\mathbf{C}$ y $\mathbf{D})$ Se observa extenso incremento de señal del cordon medular desde C2 a D10 con compromiso predominantemente en cordones laterales y posteriores.

Clínico Invitae; San Francisco, USA, en búsqueda de cambios de secuencia y deleciones/duplicaciones exónicas, identificando la variante homocigota c.1183C > T (p.Arg395Cys) clasificada como patógena. Luego de la confirmación genética, se inició tratamiento con ácido quenodesoxicólico (CDCA por su acrónimo inglés) y vitamina D.

\section{Discusión}

La prevalencia de la CXT se estima en $0.13 / 100.000$ individuos ${ }^{4}$, pero los estudios epidemiológicos disponibles sugieren un subdiagnóstico de esta enfermedad ${ }^{1,7}$. Existe una variación considerable en las características clínicas, incluyendo la ausencia de xantomas tendinosos, y edad de inicio de esta enfermedad; debido a esta variabilidad, el reconocimiento de la CXT durante la etapa inicial de la enfermedad puede ser un gran desafío $^{2}$, asociándose a un retraso diagnóstico de
16 años en promedio ${ }^{1}$.

Antiguamente se creía que la presencia de xantomas tendíneos y el compromiso neurológico eran las claves para el diagnóstico, Sin embargo, en el 2000, Verrips et al. publicaron una serie de 32 casos donde $59 \%$ se presentaba sin xantomas tendíneos en el momento del diagnóstico y $50 \%$ presentaba antecedente de diarrea crónica, proponiendo que los xantomas tendíneos son manifestaciones tardías de la enfermedad y que la diarrea, tanto en adultos como en niños, es un elemento clave en el diagnóstico ${ }^{6}$, Para el diagnóstico precoz, más allá de la prevalencia de las manifestaciones clínicas, cobra importancia la distribución por edad de las manifestaciones clínicas. En el 2018, Wong et al. llevaron a cabo una revisión de la literatura que reunió 194 casos de CTX, e intentaron calcular la probabilidad de experimentar las distintas manifestaciones clínicas de la CXT según edad, sin embargo, en muchos de los casos reportados no estaba registrada la edad 
de aparición de los síntomas ${ }^{8}$. Nuestra paciente no presentaba xantomas tendíneos visibles al examen físico, probablemente por la edad en la que consultó, pero sí presentaba la mayoría de las características clínicas clásicas de la CXT: diarrea crónica, cataratas bilaterales, colelitiasis, osteoporosis, signos piramidales y cerebelosos; a pesar de esto, el diagnóstico demoró casi dos décadas en confirmarse. La diarrea crónica desde la infancia, como en nuestro caso, puede ser la primera manifestación y debe alertar al clínico ${ }^{4}$. Los signos piramidales y cerebelosos están presentes casi invariablemente en la tercera y cuarta década de la vida. Sin embargo, la ausencia de compromiso cerebeloso tampoco descarta el diagnóstico, ya que existe una forma espinal en la que la paraparesia espástica puede ser la manifestación clínica principal $^{9,10}$.

Los hallazgos radiológicos de la CXT incluyen un incremento de señal bilateral y casi simétrico en sustancia blanca cerebelosa, periventricular, ganglios basales, núcleos dentados y tronco encefálico, así como atrofia cerebelosa y cerebral ${ }^{11}$; el compromiso de la medula espinal muestra anormalidad en los cordones laterales y dorsales, en nuestra paciente las alteraciones radiológicas fueron esenciales para el diagnóstico. Estos hallazgos imageneológicos se correlacionan histopatológicamente con múltiples hendiduras en los cristales lípidícos acumulados, macrófagos perivasculares, pérdida neuronal, desmielinización y astrocitosis reactiva a nivel encefálico, y a nivel medular desmielinización y gliosis en los tractos largos espinales s,11,12. $^{2}$.

Las anormalidades bioquímicas incluyen una concentración plasmática de colestanol cinco a diez veces mayor que la normal y una concentración elevada de alcoholes biliares en plasma y orina ${ }^{1}$. El colestanol debe medirse en ausencia de tratamiento con ácidos biliares, estatinas o corticosteroides. Los esteroides pueden reducir el colestanol en plasma al inducir la actividad residual de CYP27A1 o la eliminación de colestanol ${ }^{13}$.

El diagnóstico genético se establece con la identificación de variantes patogénica en el gen CYP27A1. Más de 90\% de las variantes patogénica descritas han sido detectadas mediante análisis de secuencia del gen CYP27A1, por lo tanto, este es el primer método diagnóstico a realizar ${ }^{4}$. Si el nivel de sospecha clínico es alto, algunos autores sugieren realizar el estudio genético sin medir los niveles de colestanol previamente ${ }^{2}$, como se hizo en este caso. Lo anterior adquiere importancia si consideramos el costo y disponibilidad de estos exámenes. La variante patógena homocigota c.1183C $>$ T (p.Arg395Cys), encontrada en esta paciente, reemplaza arginina con cisteína en el codón 395 de la proteína CYP27A1 (p.Arg395Cys). Esta variante se ha reportado como homocigótica o en combinación con otra variante de CYP27A1, en otros individuos afectados ${ }^{14-17}$. Entre los casos reportados con esta variante destaca otro paciente chileno, que además de CXT se presentó con hipercolesterolemia familiar por mutación del gen LDLR, describiendo una progresión de xantomatosis y aterosclerosis más grave y en ausencia de manifestaciones neurológicas. Es interesante destacar que esta variante tiene una alta prevalencia en España ${ }^{16}$. Otros reportes de CXT en Chile reflejan la variabilidad clínica de esta enfermedad: un caso con presentación típica asociado a xantomas tendíneos ${ }^{18}$, otro asociado a macrocefalia y dismorfias faciales, y otro sin xantomas tendíneos visibles ${ }^{19}$.

El diagnóstico diferencial de los xantomas tendíneos se reduce a la sitosterolemia y la hipercolesterolemia familiar, que no comparten las cataratas, ataxia o deterioro cognitivo de la $\mathrm{CXT}^{20,21}$. Sin embargo, la asociación de cataratas de inicio temprano y compromiso neurológico no es exclusiva de la CXT, es muy frecuente en los individuos con distrofia miotónica tipo I, seguidos por la CXT(22), la galactosemia, que también presenta diarrea e ictericia como la CTX, y el síndrome de Marinesco-Sjögren ${ }^{2}$.

La búsqueda dirigida de compromiso cardiovascular es fundamental, la aterosclerosis prematura y las enfermedades cardiovasculares se pueden presentar a pesar de un control adecuado de los niveles séricos de colesterol ${ }^{1}$. Por otro lado, se recomienda realizar estudio electrofisiólogico en búsqueda de neuropatía periférica y densitometría ósea por el riesgo de osteoporosis ${ }^{4}$.

El tratamiento de la CTX incluye terapia de reemplazo con CDCA y manejo de complicaciones (e.g. cirugía cataratas, colecistectomía, vitamina D para la osteoporosis) ${ }^{1}$. Al ser una enfermedad poco frecuente, no existen ensayos clínicos que comprueben los beneficios del tratamiento con CDCA, pero la evidencia disponible sugiere que un inicio precoz del tratamiento puede detener la progresión del daño neurológico y sistémico en estos pacientes ${ }^{23,24}$. 


\section{Referencias}

1. Nie S, Chen G, Cao X, Zhang Y. Cerebrotendinous xanthomatosis: a comprehensive review of pathogenesis, clinical manifestations, diagnosis, and management. Orphanet J Rare Dis 2014; 9: 179.

2. Mignarri A, Gallus GN, Dotti MT, Federico A. A suspicion index for early diagnosis and treatment of cerebrotendinous xanthomatosis. J Inherit Metab Dis 2014; 37 (3): 421-9.

3. Salen G, Steiner RD. Epidemiology, diagnosis, and treatment of cerebrotendinous xanthomatosis (CTX). J Inherit Metab Dis 2017; 40 (6): 771-81.

4. Federico A, Dotti MT, Gallus GN. Cerebrotendinous Xanthomatosis. In: Adam MP, Ardinger HH, Pagon RA, Wallace SE, Bean LJH, Stephens K, et al., editors. GeneReviews $^{\circledR}$. Seattle (WA) 2003.

5. Preiss Y, Santos JL, Smalley SV, Maiz A. [Cerebrotendinous xanthomatosis: physiopathology, clinical manifestations and genetics]. Rev Med Chile 2014; 142 (5): 616-22.

6. Verrips A, van Engelen BG, Wevers RA, van Geel BM, Cruysberg JR, van den Heuvel LP, et al. Presence of diarrhea and absence of tendon xanthomas in patients with cerebrotendinous xanthomatosis. Arch Neurol 2000; 57 (4): 520-4.

7. DeBarber AE, Luo J, Star-Weinstock M, Purkayastha S, Geraghty MT, Chiang JP, et al. A blood test for cerebrotendinous xanthomatosis with potential for disease detection in newborns. J Lipid Res 2014; 55 (1): 146-54.

8. Wong JC, Walsh K, Hayden D, Eichler FS. Natural history of neurological abnormalities in cerebrotendinous xanthomatosis. J Inherit Metab Dis 2018; 41 (4): 647-56.

9. Verrips A, Nijeholt GJ, Barkhof F, Van Engelen BG, Wesseling P, Luyten JA, et al. Spinal xanthomatosis: a variant of cerebrotendinous xanthomatosis. Brain. 1999; 122 (Pt 8): 1589-95.

10. Mignarri A, Rossi S, Ballerini M, Gallus GN, Del Puppo $\mathrm{M}$, Galluzzi P, et al. Clinical relevance and neurophysiological correlates of spasticity in cerebrotendinous xanthomatosis. J Neurol 2011; 258 (5): 783-90.

11. Vanrietvelde F, Lemmerling M, Mespreuve M, Crevits L, De Reuck J, Kunnen M. MRI of the brain in cerebrotendinous xanthomatosis (van Bogaert-Scherer-Epstein disease). Eur Radiol 2000; 10 (4): 576-8.

12. Barkhof F, Verrips A, Wesseling P, van Der Knaap MS, van Engelen BG, Gabreëls FJ, et al. Cerebrotendinous xanthomatosis: the spectrum of imaging findings and the correlation with neuropathologic findings. Radiology 2000; 217 (3): 869-76.

13. Siman-Tov T, Meiner V, Gadoth N. Could steroids mask the diagnosis of cerebrotendinous xanthomatosis? J Neurol Sci 2006; 243 (1-2): 83-6.

14. Szlago M, Gallus GN, Schenone A, Patino ME, Sfaelo Z, Rufa A, et al. The first cerebrotendinous xanthomatosis family from Argentina: a new mutation in CYP27A1 gene. Neurology 2008; 70 (5): 402-4.

15. Lionnet C, Carra C, Ayrignac X, Levade T, Gayraud D, Castelnovo G, et al. [Cerebrotendinous xanthomatosis: a multicentric retrospective study of 15 adults, clinical and paraclinical typical and atypical aspects]. Rev Neurol (Paris) 2014; 170 (6-7): 445-53.

16. Huijgen R, Stork AD, Defesche JC, Peter J, Alonso R, Cuevas A, et al. Extreme xanthomatosis in patients with both familial hypercholesterolemia and cerebrotendinous xanthomatosis. Clin Genet 2012; 81 (1): 24-8.

17. Pilo-de-la-Fuente B, Jiménez-Escrig A, Lorenzo JR, Pardo J, Arias M, Ares-Luque A, et al. Cerebrotendinous xanthomatosis in Spain: clinical, prognostic, and genetic survey. Eur J Neurol 2011; 18 (10): 1203-11.

18. Filippi J, Irarrazaval S, Peredo P, Mellado P. [Cerebrotendinous xanthomatosis: report of one case]. Rev Med Chile 2009; 137 (6): 815-20.

19. Smalley SV, Preiss Y, Suazo J, Vega JA, Angellotti I, Lagos CF, et al. Novel splice-affecting variants in CYP27A1 gene in two Chilean patients with Cerebrotendinous Xanthomatosis. Genet Mol Biol 2015; 38 (1): 30-6.

20. Youngblom E, Pariani M, Knowles JW. Familial Hypercholesterolemia. In: Adam MP, Ardinger HH, Pagon RA, Wallace SE, Bean LJH, Stephens K, et al., editors. GeneReviews $^{\circledR}$. Seattle (WA) 2014.

21. Merkens LS, Myrie SB, Steiner RD, Mymin D. Sitosterolemia. In: Adam MP, Ardinger HH, Pagon RA, Wallace SE, Bean LJH, Stephens K, et al., editors. GeneReviews ${ }^{\circledR}$. Seattle (WA) 2013.

22. Cruysberg JR. Cerebrotendinous xanthomatosis: juvenile cataract and chronic diarrhea before the onset of neurologic disease. Arch Neurol 2002; 59 (12): 1975.

23. Mondelli M, Sicurelli F, Scarpini C, Dotti MT, Federico A. Cerebrotendinous xanthomatosis: 11-year treatment with chenodeoxycholic acid in five patients. An electrophysiological study. J Neurol Sci 2001; 190 (1-2): 29-33.

24. Luyckx E, Eyskens F, Simons A, Beckx K, Van West D, Dhar M. Long-term follow-up on the effect of combined therapy of bile acids and statins in the treatment of cerebrotendinous xanthomatosis: a case report. Clin Neurol Neurosurg 2014; 118: 9-11. 